\title{
ATP-Liganded Form of Aspartate Transcarbamoylase, the Logical Regulatory Target for Allosteric Control in Divergent Bacterial Systems
}

\author{
J. R. WILD, ${ }^{*}$ J. L. JOHNSON, AND S. J. LOUGHREY \\ Department of Biochemistry and Biophysics, Texas A \& M University System, College Station, Texas 77843
}

Received 15 July 1987/Accepted 30 September 1987

\begin{abstract}
In Escherichia coli, the mechanism for regulatory control of aspartate transcarbamoylase is clear; CTP allosterically inhibits catalysis in direct competition with ATP. However, both CTP and ATP may be activators or may have no effect on aspartate transcarbamoylases from other enteric bacteria. A common regulatory logic observed was that the ATP-activated enzymes were rendered less active as the result of competition with CTP, regardless of the independent effects.
\end{abstract}

The de novo biosynthesis of pyrimidine nucleotides is regulated by a series of enzymatic, genetic, and physiological control systems which integrate production with intracellular nucleotide pool levels $(4,12,15)$. These metabolic controls coordinate the synthesis and utilization of carbamoyl phosphate by arginine and pyrimidine biosynthetic pathways. The integrated regulatory logic of purine, pyrimidine, and arginine biosyntheses in Escherichia coli and Salmonella typhimurium revolves around the allosteric control of aspartate transcarbamoylase (EC 2.1.3.2; ATCase), the first enzyme unique to pyrimidine biosynthesis $(8,11$, 14). In those bacteria, ATCase is feedback inhibited by CTP and activated by ATP $(8,15)$. However, the ATCases from other enteric bacteria may be activated by CTP or ATP or both $(5,7,18,19)$ or may have no apparent heterotropic response to CTP or ATP whatsoever $(2,7,19)$. The enzymes from members of various tribes of the family Enterobacteriaceae are similar in molecular architecture in that they are synthesized as distinct regulatory and catalytic subunits which are assembled into dodecameric holoenzymes in vivo $(5,9,16)$. The dodecamer is organized such that two independently functional catalytic trimers are associated through three regulatory dimers, $2\left(c_{3}\right): 3\left(r_{2}\right)$; to establish homotropic communications between active sites $(9,11)$. The architectural similarities have made it possible to form hybrid enzymes from regulatory subunits of one species and catalytic subunits of another $(5,14,16)$. These hybrids suggest that the regulatory subunits prescribe the allosteric responses of the holoenzyme (5). Despite the structural similarities, the various "class B" ATCases (2) are dramatically different in catalytic and regulatory properties. While all the enzymes are characterized by homotropic kinetics toward aspartate, the concentration required for half-maximal activity can vary from 3 to $30 \mathrm{mM}(5,7,18,19)$. It has been possible to identify seven regulatory classes of these enzymes on the basis of allosteric and substrate requirements, and these classes generally conform to tribal classifications of the bacteria $(5,7,19)$. The enzymatic characteristics of the ATCases from the bacterial strains used in these studies are summarized in Table 1.

The allosteric effector responses of selected ATCases were compared with those of enzymes overproduced in $E$. coli EK1104 (13) transformed with pyrBI operons from

\footnotetext{
* Corresponding author.
}

selected members of the family Enterobacteriaceae (pPBh105-Ec, E. coli; pPBh101-Sm, Serratia marcescens; pPBh103-Pv, Proteus vulgaris; pPBh103-Eh, Erwinia herbicola) (13). Holoenzyme forms of ATCase were separated from independent catalytic trimers by chromatography over Sephadex DEAE A-50 according to the procedures of Yang et al. (20). The saturation kinetics of the allosteric effector responses were measured in the presence of 0 to $5 \mathrm{mM}$ nucleotide at aspartate concentrations approximating onehalf of the $S_{0.5}$ for each enzyme (Table 1). A saturating concentration of carbamoyl phosphate $(5 \mathrm{mM})$ was used. Enzyme assays were performed in $10 \mathrm{mM}$ Tris, bis-Tris, and CAPS [3-(cyclohexylamino)-1-propanesulfonic acid] buffer (pH 7.0) to avoid nonspecific phosphate effects (see Fig. 1 to 3 ) as described previously $(6,19)$. The allosteric responses of the ATCases to CTP and ATP are shown in Fig. 1 and 2. From these data, it was possible to estimate the relative binding of the allosteric effectors to each enzyme. The CTP concentrations required to provide $50 \%$ inhibition were similar for each affected enzyme $(0.1,0.3$, and $0.1 \mathrm{mM}$ for enzymes produced by $E$. coli, $P$. vulgaris, and $S$. marcescens, respectively) and demonstrated a relatively tight binding for CTP (Fig. 1). The analogous evaluation of saturation by ATP (Fig. 2) revealed that ATP did not bind as tightly to the enzyme, and the relative saturation varied from 0.6 to 0.8

TABLE 1. Selected enterobacterial strains and their ATCases

\begin{tabular}{lll}
\hline \multicolumn{1}{c}{ Species } & \multicolumn{1}{c}{ Strain $^{a}$} & \multicolumn{1}{c}{ Allosteric response $^{b}$} \\
\hline E. coli K-12 & CGSC 4255 & CTP $^{-}$, ATP $^{+}(5 \mathrm{mM})$ \\
$E$. herbicola & TAMU 177 & CTP $^{0}$, ATP \\
$P$. vulgaris & ATCC 13315 & CTP $^{+}$, ATP $^{+}(30 \mathrm{mM})$ \\
S. marcescens HY & ATCC 8195 & CTP $^{+}$, ATP $^{+}(20 \mathrm{mM})$ \\
$Y$. enterocolitica & CDC 175 & CTP $^{0}$, ATP $^{0}(5 \mathrm{mM})$ \\
$Y$. fredriksenii & CDC 867 & CTP $^{0}$, ATP $^{0}(5 \mathrm{mM})$ \\
$Y$. intermedia & CDC 48 & CTP $^{-}$, ATP $^{+}(5 \mathrm{mM})^{c}$ \\
$Y$. kristensenii & CDC 1475 & CTP $^{0}$, ATP $^{0}(5 \mathrm{mM})$ \\
\hline
\end{tabular}

${ }^{a}$ CGSC, E. coli Genetic Stock Center, Yale University, New Haven, Conn.; TAMU, Texas A \& M University (strain supplied by Bill Foster); ATCC, American Type Culture Collection, Rockville, Md.; CDC, Centers for Disease Control, Atlanta, $\mathrm{Ga}$.

${ }^{b} \mathrm{CTP}^{-}$, Inhibition by $2 \mathrm{mM}$ CTP; CTPs, slight inhibition by CTP; $\mathrm{CTP}^{+}$or $\mathrm{ATP}^{+}$, stimulation by CTP or ATP, respectively; CTP ${ }^{0}$ or $\mathrm{ATP}^{0}$, no effect by CTP or ATP, respectively. The aspartate concentration used for allostericresponse determination is indicated parenthetically; each value represents approximate $S_{0.5}$ values estimated from previous studies $(5,8,19)$.

c UTP inhibition was observed with this enzyme. 


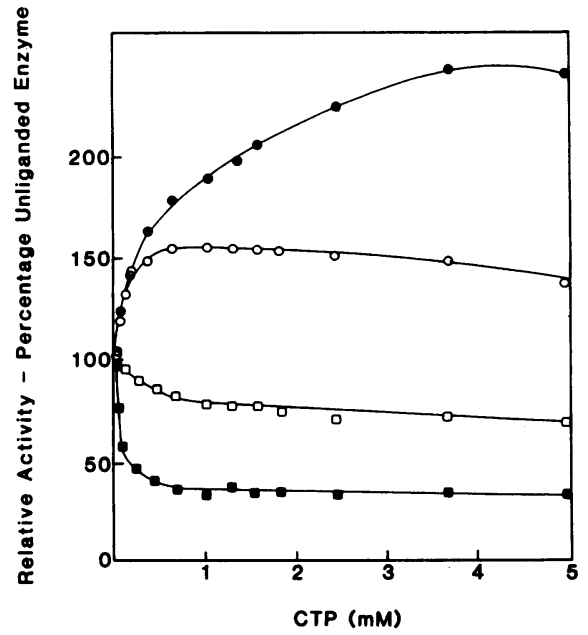

FIG. 1. Effect of CTP on activity of ATCases from selected bacterial sources. Standard assay conditions involved the use of a saturating concentration of carbamoyl phosphate $(2 \mathrm{mM})$ and concentrations of aspartate which approximated the $S_{0.5}$ of each enzyme: $2.5 \mathrm{mM}$ for the $E$. coli enzyme ( $\boldsymbol{\square}) ; 15 \mathrm{mM}$ for the $P$. vulgaris enzyme (O); $3 \mathrm{mM}$ for the $S$. marcescens enzyme $(O)$; and $1.5 \mathrm{mM}$ for the $E$. herbicola enzyme ( $\square$ ).

mM (four- to fivefold higher than that for CTP). The ATCase of $E$. herbicola did not respond to ATP and showed a low level of inhibition by CTP (15 to 20\%).

When the effects of CTP were determined for the various ATCases in the presence of a saturating concentration of ATP $(2 \mathrm{mM})$, it was found that CTP induced a significant reduction of activity regardless of the independent effect of CTP on the native enzymes (Fig. 3). For example, the ATCase of $P$. vulgaris was able to use CTP as an inhibitor (45\%) of the ATP-activated enzyme, even though CTP activated the enzyme by itself. The concentrations of CTP required to produce the maximal effect were higher in the presence of ATP than they were in its absence $(0.8,1.2$, and $1.2 \mathrm{mM}$ for the enzymes produced by $E$. coli, $S$. marcescens, and $P$. vulgaris, respectively). Although the inhibition of the ATCase of $E$. herbicola did not change overall, two- to

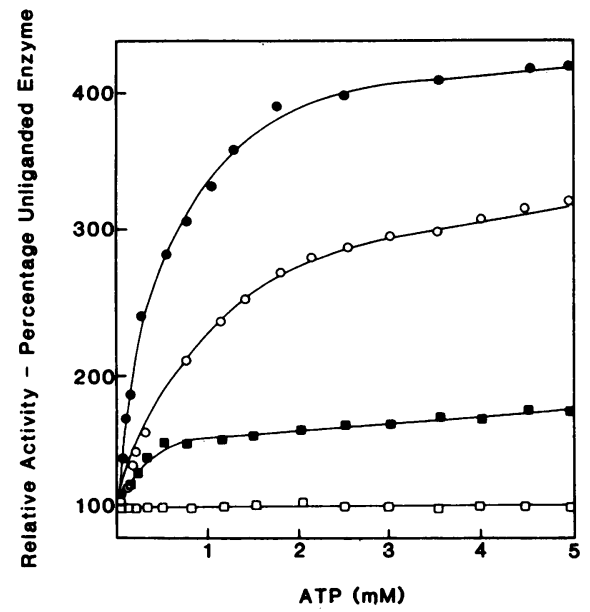

FIG. 2. Effect of ATP on activity of ATCases from selected bacterial sources. Assay conditions were identical to those described in the legend to Fig. 2. The enzymes were from $E$. coli $(\square)$, $P$. vulgaris $(\bigcirc), S$. marcescens $(\bigcirc)$, and $E$. herbicola $(\square)$.

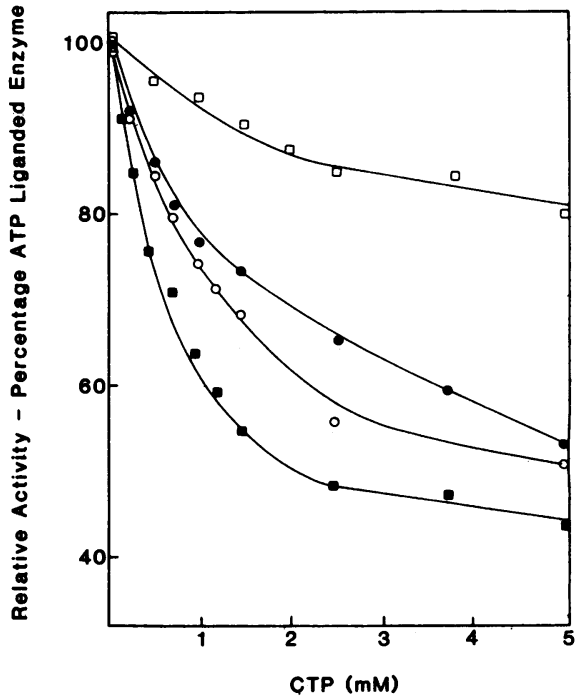

FIG. 3. Effect of CTP on activity of ATP-activated ATCases from selected bacterial sources. The catalytic activities of several ATCases were determined under conditions in which the enzyme was preincubated with $2 \mathrm{mM}$ ATP and assayed in the presence of various concentrations of CTP $(0$ to $5 \mathrm{mM})$. The enzymes were from E. coli $(\mathbb{\square}), P$. vulgaris $(\Theta), S$. marcescens $(O)$, and $E$. herbicola (口).

threefold-higher concentrations of CTP were required to develop maximal effects in the presence of ATP. With all the enzymes examined, lowering the aspartate concentration accentuated the differences between the ATP- and the CTPactivated enzymes (data not shown).

To evaluate the physiological significance of these observations, nucleotide pools were measured by the method of Chen et al. (3) and quantitated by comparative integration with known standard samples (Table 2 ). The various enteric strains differred slightly in the levels of endogenous nucleotide pools and could be divided into three groups: (i) $E$. coli and Yersinia intermedia 48 had equivalent pool levels; (ii) Yersinia fredriksenii pools were approximately $50 \%$ higher than those of $E$. coli; and (iii) the nucleotide pools of $P$. vulgaris, $S$. marcescens, $E$. herbicola, Yersinia enterocolitica, and Yersinia kristensenii were 30 to $70 \%$ lower than those of $E$. coli. Nonetheless, these relative pool sizes were in the millimolar range, and they remained relatively con-

TABLE 2. Nucleotide pools in selected members of the Enterobacteriaceae ${ }^{a}$

\begin{tabular}{lllllc}
\hline \multirow{2}{*}{\multicolumn{1}{c}{ Species }} & \multicolumn{5}{c}{ Average nucleotide pools $^{b}$} \\
\cline { 2 - 6 } & CTP & UTP & ATP & GTP & UTP/CTP \\
\hline E. coli & 1.09 & 1.43 & 3.00 & 1.84 & 1.3 \\
$E$. herbicola & 0.55 & 1.21 & 1.73 & 1.24 & 2.2 \\
$P$. vulgaris & 0.63 & 0.95 & 2.65 & 1.63 & 1.5 \\
S. marcescens & 0.92 & 1.07 & 1.64 & 1.02 & 1.2 \\
$Y$. enterocolitica & 0.33 & 0.53 & 1.16 & 0.41 & 1.6 \\
$Y$. fredriksenii & 1.53 & 3.25 & 5.62 & 3.10 & 2.1 \\
$Y$. intermedia & 1.15 & 2.08 & 3.53 & 1.48 & 1.8 \\
$Y$. kristensenii & 0.64 & 1.05 & 2.19 & 1.25 & 1.6 \\
\hline
\end{tabular}

${ }^{a}$ Grown in minimal medium plus $0.1 \%$ Casamino Acids.

${ }^{b}$ Measured as micromoles of nucleotide per gram (dry weight). Values are averages of three to five independent culture measurements. Standard deviations were approximately 5 to $20 \%$ of determined pool values.

c Ratios calculated from nucleotide pool averages. 
stant regardless of the type of medium in which the strains were grown (J. L. Johnson, M.S. thesis, Texas A \& M University, College Station, 1984). Despite the variation in the absolute levels of nucleotide pools, a remarkable consistency was observed in the ratios of the nucleotide pools. Each of the bacterial species maintained a UTP/CTP ratio between 1.3 and 2.2, which increased 20 to $60 \%$ upon growth in defined medium supplemented with $50 \mu \mathrm{g}$ of uracil per $\mathrm{ml}$ (unpublished observation).

An evaluation of alternate regulatory parameters was undertaken by examining the expression and apparent allosteric control of carbamoyl phosphate synthetase (EC 6.3.5.5). Each of the carbamoyl phosphate synthetases examined (strains listed in Fig. 1) were inhibited 60 to $70 \%$ by UMP, while ornithine stimulated activities 100 to $300 \%$. These characteristics are comparable to the regulatory controls observed for $E$. coli $(1,4)$. Similarly, there were no apparent differences between the various ornithine transcarbamoylases and their $E$. coli counterpart. The ornithine transcarbamoylases were not subject to allosteric control by purine or pyrimidine nucleotides. Therefore, it appears that the regulatory differences in the ATCases are not corrected or accommodated by changes in carbamoyl phosphate synthetase or ornithine transcarbamoylase.

In $E$. coli, the levels of intracellular pools of aspartate are estimated to be between 2 and $5 \mathrm{mM}$, approximating the concentrations required for half-maximal activity of its ATCase $(8,11,15)$. Since catalytic activity is most sensitive to allosteric effectors at substrate concentrations below their $S_{0.5} \mathrm{~s}$, nucleotide effectors would be expected to have their greatest impact at those lower substrate ranges. Furthermore, the nucleotide effectors, ATP and CTP, produce their maximal allosteric controls at concentrations approximating intracellular pool levels (Fig. 1 to 3; Table 2). To understand the regulatory logic of ATCase, one must always consider the competition of ATP and CTP for the same allosteric binding sites $(10,11,17)$, whether the two nucleotides produce different heterotropic responses $(8,17)$ or not $(2,7$, 19). As observed in these studies, ATP-activated catalysis was reduced in the presence of CTP regardless of whether CTP functioned independently as an inhibitor or as an activator or had no effect on the enzyme. It is not clear why other oligomeric ATCases (e.g., the ATCase of $Y$. enterocolitica) (7) do not appear to respond to the presence of any purine and pyrimidine nucleotides. These enzymes have regulatory polypeptides and possess homotropic kinetics relative to aspartate concentrations, yet their regulatory logic remains cryptic $(5,7,19$; Wild et al., unpublished observations)

This research was supported by the National Science Foundation (DMB-8703732), the Robert A. Welch Foundation (A-915), and the Texas Agricultural Experiment Station.

\section{LITERATURE CITED}

1. Bagnara, A. S., and L. R. Finch. 1974. The effects of bases and nucleosides on the intracellular contents of nucleotides and 5-phosphoribosyl 1-pyrophosphate in Escherichia coli. Eur. J.
Biochem. 41:421-430.

2. Bethell, M. A., and M. E. Jones. 1969. Molecular size and feedback-regulation characteristics of bacterial aspartate transcarbamylases. Arch. Biochem. Biophys. 134:352-365.

3. Chen, S. C., P. R. Brown, and D. M. Rosie. 1977. Extraction procedures for use prior to HPLC nucleotide analysis using microparticle chemically bonding packings. J. Chromatogr. Sci. 15:218-221.

4. Cunin, R. 1980. Regulation of arginine biosynthesis in prokaryotes, p. 53-79. In R. M. Somerville, (ed.), Amino acids: biosynthesis and genetic regulation, Academic Press, Inc., New York.

5. Foltermann, K. F., D. A. Beck, and J. R. Wild. 1986. In vivo formation of hybrid aspartate transcarbamoylases from native subunits of divergent members of the family Enterobacteriaceae. J. Bacteriol. 167:285-290.

6. Foltermann, K. F., M. S. Shanley, and J. R. Wild. 1984. Assembly of the aspartate transcarbamoylase holoenzyme from transcriptionally independent catalytic and regulatory cistrons. J. Bacteriol. 157:891-898.

7. Foltermann, K. F., J. R. Wild, D. L. Zink, and G. A. O'Donovan. 1981. Regulatory variance of aspartate transcarbamoylase among strains of Yersinia enterocolitica and Yersinia enterocolitica-like organisms. Curr. Microbiol. 6:43-47.

8. Gerhart, J., and A. B. Pardee. 1962. The enzymology of control by feedback inhibition. J. Biol. Chem. 237:891-896.

9. Gerhart, J., and H. K. Schachman. 1965. Distinct subunits for the regulation and catalytic activity of aspartate transcarbamylase. Biochemistry 4:1054-1062.

10. Honzatko, R. B., and W. N. Lipscomb. 1982. Interactions of phosphate ligands with Escherichia coli aspartate carbamoyltransferase in the crystalline state. J. Mol. Biol. 160:265-286.

11. Kantrowitz, E. R., S. C. Pastra-Landis, and W. N. Lipscomb. 1980. E. coli aspartate transcarbamylase, part I: catalytic and regulatory functions. Trends Biochem. Sci. 5:124-128.

12. Makoff, A. J., and A. Radford. 1978. Genetics and biochemistry of carbamoyl phosphate biosynthesis and its utilization in the pyrimidine biosynthetic pathway. Microbiol. Rev. 42:307-328.

13. Nowlan, S. F., and E. R. Kantrowitz. 1985. Superproduction and rapid purification of Escherichia coli aspartate transcarbamylase and its catalytic subunit under extreme derepression of the pyrimidine pathway. J. Biol. Chem. 260:14712-14716.

14. O'Donovan, G. A., H. Holoubek, and J. C. Gerhart. 1972. Regulatory properties of intergeneric hybrids of aspartate transcarbamylase. Nature (London) New Biol. 238:264-266.

15. O'Donovan, G. A., and J. Neuhard. 1970. Pyrimidine metabolism in microorganisms. Bacteriol. Rev. 34:278-343.

16. Shanley, M. S., K. F. Foltermann, G. A. O'Donovan, and J. R. Wild. 1984. Properties of hybrid aspartate transcarbamoylase formed with native subunits from divergent bacteria. J. Biol. Chem. 259:12672-12677.

17. Wedler, F. C., and F. J. Gasser. 1974. Modes of modifier action in E. coli aspartate transcarbamylase. Arch. Biochem. Biophys. 163:69-78.

18. Wild, J. R., W. L. Belser, and G. A. O'Donovan. 1976. Unique aspects of the regulation of the aspartate transcarbamylase of Serratia marcescens. J. Bacteriol. 128:766-775.

19. Wild, J. R., K. F. Foltermann, and G. A. O'Donovan. 1980. Regulatory divergence of aspartate transcarbamoylases within the Enterobacteriaceae. Arch. Biochem. Biophys. 201:506-517.

20. Yang, Y. R., M. W. Kirschner, and H. K. Schachman. 1978. Aspartate transcarbamoylase (Escherichia coli): preparation of subunits. Methods Enzymol. 51:35-41. 